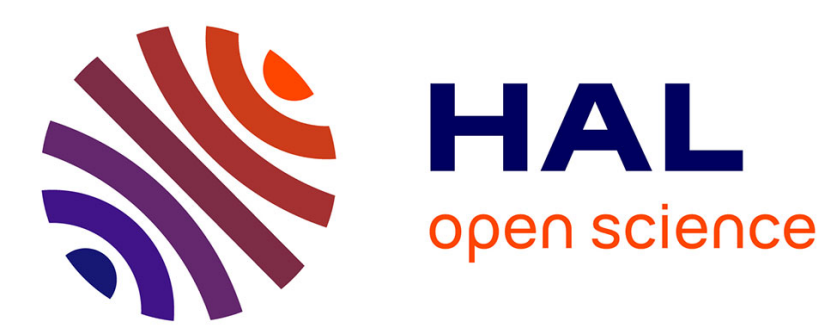

\title{
Renouveau des jardins potagers entre milieux urbain et rural ?
}

Manon Gallien, Marjolaine Boitard, Claire Delfosse

\section{To cite this version:}

Manon Gallien, Marjolaine Boitard, Claire Delfosse. Renouveau des jardins potagers entre milieux urbain et rural ?. Pour: enquêtes et témoignages, 2012, Alimentation et territoires, 215-216 (3), pp.321-332. 10.3917/pour.215.0321 . halshs-01633996

\section{HAL Id: halshs-01633996 \\ https://shs.hal.science/halshs-01633996}

Submitted on 8 Feb 2018

HAL is a multi-disciplinary open access archive for the deposit and dissemination of scientific research documents, whether they are published or not. The documents may come from teaching and research institutions in France or abroad, or from public or private research centers.
L'archive ouverte pluridisciplinaire HAL, est destinée au dépôt et à la diffusion de documents scientifiques de niveau recherche, publiés ou non, émanant des établissements d'enseignement et de recherche français ou étrangers, des laboratoires publics ou privés. 


\section{Manon GALLIEN}

Doctorante, Laboratoire d'études rurales (LER), Université Lyon 2

\section{Marjolaine BOITARD}

Étudiante en Master 1, LER

\section{Claire DELFOSSE}

Professeure, LER, Université Lyon 2

\section{«RENOUVEAU 》 DES JARDINS POTAGERS ENTRE MILIEUX URBAIN ET RURAL?}

Même si le jardin est un espace limité par sa superficie, où l'on cultive des végétaux utiles et d'agrément, son rôle utilitaire est réhabilité. En ville comme à la campagne on redécouvre l'importance de sa fonction nourricière face à la pauvreté, à l'intérêt pour les produits sains, à l'attention portée à la biodiversité, ainsi qu'à la préservation de l'environnement. Le jardin potager revêt une multiplicité des formes, individuelles (jardin de bourg, jardin de lotissement, verger) et collectives (jardins familiaux anciennement ouvriers, jardins d'insertion, jardins pédagogiques, jardins partagés) ; les formes individuelles étant plus présentes en milieu rural et les formes collectives en ville. Nous nous sommes interrogés ici sur la transformation des jardins potagers face aux nouveaux enjeux alimentaires en croisant urbain et rural. Le rôle utilitaire du potager 


\section{Carte 1 - Répartition des jardins étudiés} en périphérie de Roanne

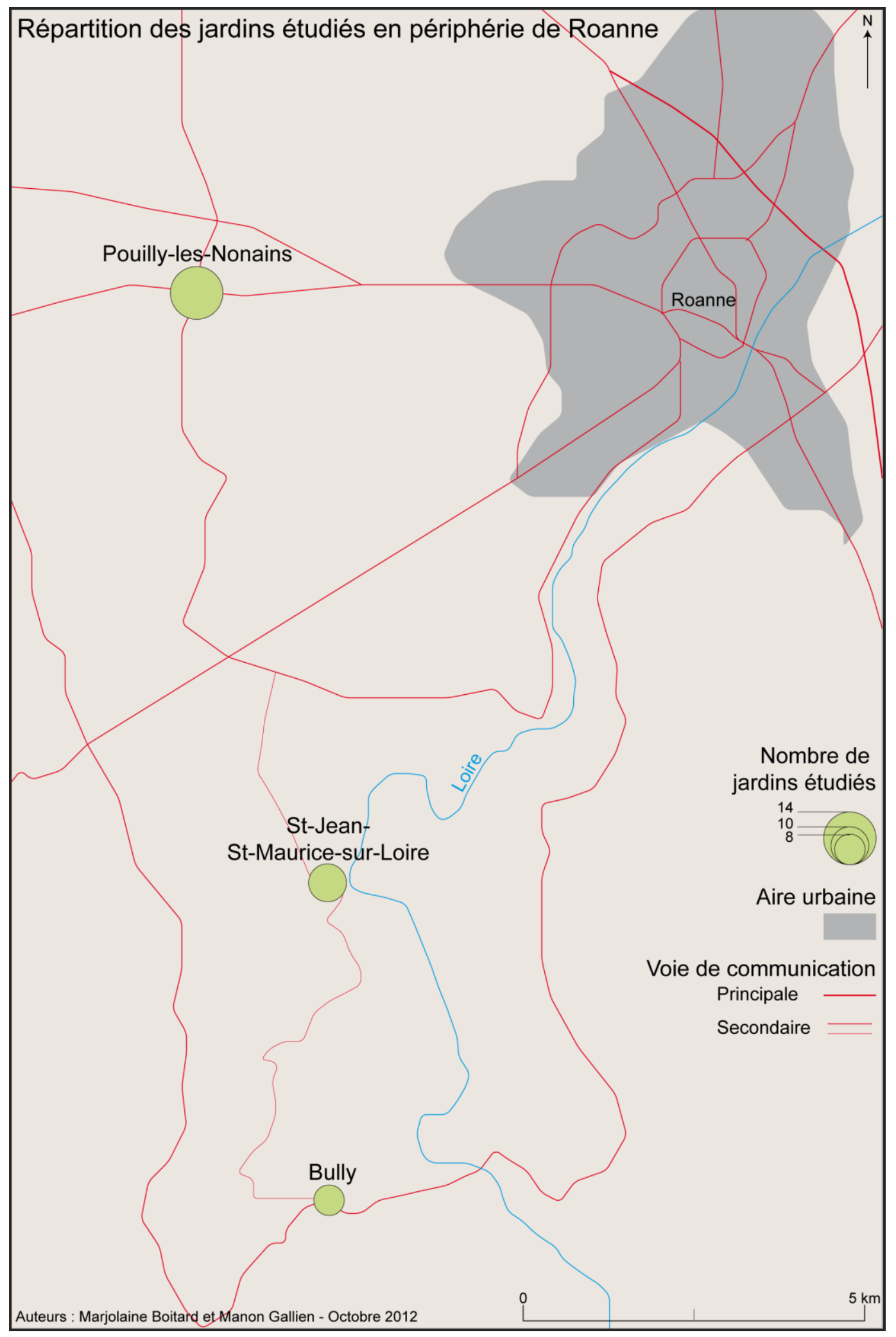

Auteurs : Marjolaine Boitard et Manon Gallien - Octobre 2012 
prend-t-il le dessus sur ses qualités récréatives ? Quel est l'impact des discours environnementalistes sur les pratiques potagères?

Cet article repose sur une étude exploratoire menée à Roanne et dans sa périphérie (département de la Loire), un espace que l'on peut considérer comme étant en crise ${ }^{1}$. II s'appuie sur des entretiens menés auprès du milieu associatif, des mairies des communes étudiées, de jardineries et de deux structures collectives à vocation nourricière et de loisirs ${ }^{2}$. Enfin, 34 enquêtes ont été réalisées auprès de jardiniers amateurs, dans les jardins individuels de trois villages, choisis pour leur degré d'éloignement avec l'aire urbaine roannaise (voir carte 1$)^{3}$.

Avant d'analyser de façon détaillée les fonctions des jardins potagers et le poids des discours sur les pratiques de jardinage, il convient de revenir sur le contexte « jardinier » spécifique de Roanne et du Roannais.

\section{Un contexte historique et social favorisant le développement de structures associatives autour des jardins}

Le Roannais est considéré comme un territoire industriel en crise. Cette dernière touche tout particulièrement Roanne, ville moyenne située au Nord du département de la Loire, et se traduit par une forte diminution de la population (55 198 habitants en 1975 et 35936 en 2008) accompagnée d'un vieillissement et d'un appauvrissement. Dans ce contexte l'économie sociale et solidaire (ESS) joue un rôle primordial. La crise suscite de la part de la ville des politiques sociales importantes, ainsi qu'une volonté politique forte de changer l'image de la ville. L'espace rural avoisinant lui aussi est touché par la crise, toutefois quelques communes de la côte Roannaise, où l'on trouve de la vigne, jouent la carte de la valorisation du patrimoine ainsi que de l'attractivité résidentielle et de loisirs. Ainsi, alors que la population de la ville de Roanne a très fortement diminué des communes périurbaines et rurales environnantes ont vu leur nombre d'habitants augmenter.

\footnotetext{
${ }^{1}$ Cette enquête exploratoire a été réalisée à l'occasion d'un travail de terrain conduit avec des étudiants de Master 1 de géographie de l'Université de Lyon2 en 2011et 2012. Chacune de ces années un dossier individuel sur les jardins a été réalisé, des dossiers que nous avons très largement mobilisés : Lois Besançon sur les jardins collectifs à Roanne et celui de Marjolaine Boitard sur le renouveau des jardins. Cet article s'inscrit également dans le cadre d'une thèse qui débute réalisée par Manon Gallien sur les jardins potager en milieu rural.

${ }_{2}^{2}$ Les responsables du jardin pédagogique de la classe de CE1 et du projet de verger pédagogique de Pouilly-lesNonains ont été rencontrés.

${ }^{3}$ II s'agit des communes de Pouilly-les-Nonains, à $8 \mathrm{~km}$ de Roanne, commune périurbaine de 1884 habitants (Insee 2009), dont la démographie augmente depuis 8 ans; de Saint-Jean-Saint-Maurice-sur-Loire, village de 1136 habitants au label « villages et cités de caractère », situé à $12 \mathrm{~km}$ de Roanne, qui connait lui aussi une lente augmentation de sa population depuis 1975 et enfin de Bully, commune de 391 habitants3 à 20km de Roanne.
} 


\section{Une importance ancienne renouvelée des jardins collectifs à Roanne}

La ville de Roanne, comme les environs, compte un grand nombre d'associations qui ont pour vocation «les jardins». Pour les plus anciennes on trouve les jardins familiaux particulièrement importants à Roanne et des jardins portés aussi par des associations relevant du catholicisme social.

Les jardins collectifs sont étroitement liés à l'histoire de Roanne, comme à celle du département de la Loire. Roanne accueille ses premiers jardins en 1920 et en 1942 ils comptent 500 adhérents. Le caractère industriel de la ville favorise ce développement: des industries mettent à disposition des terrains. En 1954 on compte 19 jardins ouvriers à Roanne ${ }^{4}$. Si la crise industrielle entraîna la fermeture de nombreuses usines, les jardins, eux, subsistèrent. Certes leur attrait et leur nombre diminue dans les années 1970, mais depuis 1996, Roanne enregistre une nouvelle hausse de demande d'attribution de parcelles. Face à ce nouvel intérêt, la municipalité signe en 1997, une charte permettant de définir les objectifs des jardins collectifs ; une charte modernisée en 2011 avec le changement de majorité et qui permet d'intégrer les jardins partagés qui ont vu le jour à Roanne dans le courant des années 2009-2010. Cette charte comporte cinq objectifs : «lien social et intégration dans la vie de la ville »; « des lieux d'échanges autour du jardinage » ; «pour une alimentation saine »; «produire pour une alimentation saine à coût faible »; «pour la protection de l'environnement » et enfin « pour la protection de l'environnement ». En 2011, la ville compte dix jardins collectifs relevant de trois types de statuts : des jardins ouvriers ou familiaux constitués en association; des jardins partagés hébergés par une association et des jardins non constitués en association. Les

Tableau 1 : Répartition par profession des adhérents aux jardins familiaux en 2009

\begin{tabular}{|c|c|}
\hline Profession & Répartition en \% \\
\hline Employés & 55.0 \\
\hline Sans emploi & 22.8 \\
\hline Retraités & 8.9 \\
\hline Travailleurs manuels & 5.2 \\
\hline Cadres & 4.2 \\
\hline Professions libérales & 2.7 \\
\hline Etudiants & 0.9 \\
\hline Autres & 0.4 \\
\hline
\end{tabular}

Source : Association des jardins ouvriers et familiaux de Roanne

\footnotetext{
${ }^{4}$ La ville tend d'ailleurs à conserver le terme de jardin ouvrier alors que la loi de 1952 en fait des jardins familiaux.
} 
premiers sont au nombre de 4 et dominent largement en termes de superficie 93 hectares sur les 117,5 ha dédiés aux jardins collectifs. Ils adhérent à la fédération des jardins ouvriers de la Loire. Dans la plupart des cas, les terrains sont mis à disposition par la ville de Roanne ou par l'Etat. Dans deux des cas le propriétaire des terrains est un propriétaire privé. Mais la mise à disposition est systématiquement faite à titre gracieux. Les jardins ouvriers et familiaux visent à l'autoproduction alimentaire de fruits et légumes et concernant essentiellement une population modeste.

Les jardins indépendants sont des jardins mis en culture à l'initiative des habitants qui ne sont pas regroupés en collectif ou en association et qui occupent le domaine fluvial (bords de Loire) ou un emplacement historique de jardin ouvrier.

Les jardins partagés roannais sont au nombre de deux. Dans cette ville en crise, et où l'économie sociale et solidaire revêt une grande importance, ils ont des caractéristiques particulières, ils ne sont pas constitués en association ou en collectifs, mais sont hébergés et gérés par des associations déjà existantes : l'un a été créé à l'initiative du secours catholique et l'autre des jardins de Cocagne.

\section{Des jardins partagés : témoins de la vitalité de l'économie sociale et solidaire}

De nouvelles associations, relevant de l'ESS, se sont développées depuis une dizaine d'années, à Roanne et dans les espaces ruraux en périphérie de la ville. Elles sont porteuses de valeurs alternatives à la consommation de masse, telles que l'entraide, le respect de l'humain et de l'environnement. Leurs adhérents sont majoritairement jeunes et/ou actifs et pratiquent le potager.

Les jardins de Cocagne sont un exemple d'association militant par et pour le potager. Le plus souvent les jardins de Cocagne sont installés en milieu rural (afin de disposer de plus d'espace), mais ceux de Roanne sont les seuls à se trouver en milieu urbain (il reste d'anciens jardins ouvriers et à Roanne il n'y a pas de spéculation foncière, au contraire).

Les jardins de Cocagne Roannais ont été créés en 1999. Ils comptent 40 salariés et plus de 220 adhérents par an qui consomment sa production maraîchère. La ville de Roanne apporte un soutien important au réseau : le terrain est mis à disposition à titre gracieux, il en est de même pour les locaux ainsi que pour l'eau d'irrigation.

Outre le jardin d'insertion, les jardins de Cocagne sont à l'initiative de la création d'un deux jardins partagés de Roanne avec le «Jardin des mères et pères ». II 
Les jardins de Cocagne : Réseau de jardins maraichers en $A B$. Le premier date de 1991. Ces jardins ont pour vocation d'une part de bien nourrir en proposant des paniers hebdomadaires de légumes, et d'autre part d'insérer en employant des personnes en difficultés financières et sociales.

Kokopelli : Association fondée en 1999. Elle œuvre pour «la libération de l'humus et des semences " et l'agro-écologie. Ses membres considèrent que l'agriculture conventionnelle, avec ses semences non reproductibles et ses intrants chimiques, est un poison pour la planète. Ils ont une réserve de plus de 2200 « semences libres » de variétés anciennes ou non.

Les croqueurs de pommes: Mouvement national fondé en 1978, aujourd'hui riche de soixante associations et 7000 adhérents. II milite pour la sauvegarde de variétés fruitières régionales en voie de disparition. II implante des vergers sur des communes et organise des formations à la greffe et à la plantation.

s'inscrit dans le cadre d'une politique de sensibilisation au jardin et dans la politique de la ville de Roanne dans le quartier du parc. Les jardins de Cocagne mettent à disposition des habitants du quartier une vingtaine de parcelles de $30 \mathrm{~m}^{2}$ pour les personnes les plus démunies. La demande d'attribution est ainsi gérée par la maison de service public du quartier. Le président de la structure roannaise, souligne leur rôle vivrier puisque $70 \%$ de la population de ce quartier vit en dessous du seuil de pauvreté et «le reste, juste au-dessus ». Ces parcelles sont cultivées en agriculture biologique.

L'autre jardin partagé de Roanne, celui de l'Accueil fraternel du secours catholique de Roanne datant de 2010, peut être considéré comme un héritage du poids du catholicisme social à Roanne. Les jardiniers ne sont pas adhérents, mais inscrits comme participants auprès du FRAT et peuvent prendre part aux sessions de travail dans le jardin. L'objectif de ce jardin est de créer du lien social autour d'une activité qui n'est pas que ludique mais qui permet de « rémunérer » les participants pour le travail fourni. En effet cette activité s'assortit de la mise en place d'une monnaie alternative le Noy correspondant au temps de travail effectué au sein du FRAT et qui permet d'acheter la production ou des vêtements au centre de tri du secours catholique ou enfin de participer à des voyages organisés par FRAT. A l'inverse, les personnes donnant du temps au centre de tri de vêtements peuvent achetez des légumes produits dans les jardins. Ici, l'activité de jardinage permet donc de rompre l'isolement, mais revêt aussi un volet économique et peut procurer des aliments. 


\section{Un certain renouveau autour de la fonction nourricière entre urbain et rural}

Les milieux ruraux proches de Roanne connaissent une vague de nouveaux arrivants urbains s'installant « à la campagne ». Ces « rurbains » peuvent avoir une pratique du potager différente qui permet de dépasser l'image d'un potager nourricier, en milieu rural, réservé aux retraités. Certains rurbains et ruraux développent des potagers par nécessité et/ou conviction. Ces jardins-là sont valorisés par des associations de ruraux alternatifs ou bien par des associations à forte volonté d'insertion des populations précaires. II s'agit des jardins de Cocagne, mais aussi d'une association originale transcendant l'urbain et le rural : Entre POTE (Entreprise Paysans Ouvriers Travailleurs Equitables). Cette association est installée depuis 2007 à Roanne. Elle compte 200 adhérents à l'année ; ils sont âgés de 30 à 50 ans et habitent dans le Roannais rural (Bully, Arpheuilles, Riorges). Elle a été porteuse de nombreuses activités comme la création des AMAPs du Roannais. Sa fondation résulte de la création d'un groupement d'achat de produits issus de l'AB, en réaction à l'alimentation industrielle qui ne répondait pas à leurs valeurs. Elle est forte de valeurs respectueuses de l'humain et de l'environnement. L'association organise notamment des échanges de graines et de plants pour le potager. L'une des membres est aussi adhérente de l'association Kokopelli (voir encadré) et permet un apport de semences en $A B$ (critère essentiel à l'association). Le potager en milieu rural est considéré, en premier lieu, comme un espace nourricier, dont la réalisation s'effectue grâce à de l'entraide et une mise en réseau des savoir-faire. En second lieu, il est aussi porteur de valeurs anticonsuméristes. L'autoproduction et l'autoconsommation grâce au jardin potager sont ici vues comme un acte marginal et revendicatif.

La fonction nourricière des jardins est également ré-envisagée pour son rôle patrimonial. Le projet de verger pédagogique de Pouilly-les-Nonains est, à cet égard, significatif. II appartient à une Opération Programmée d'Amélioration des Vergers (OPAV), subventionnée par le syndicat mixte «Roannais-Pays de Rhône-Alpes ». Cette initiative de la communauté de communes de l'Ouest Roannais (CCOR) a débuté à Pouilly-les-Nonains avec l'installation de porte-greffes, en collaboration avec l'association des Croqueurs de Pomme (voir encadré). À terme, la commune devrait accueillir 100 arbres fruitiers de variétés régionales ou rares. L'entretien mené à la mairie de Pouilly soulignait le rôle de ces vergers dans la qualité de vie des habitants. Ainsi, les plantations sont ouvertes à tous et seront l'objet de cours de greffe. Par la suite, ces vergers auront pour double vocation d'offrir un cadre agréable en tant qu'espace vert et de fournir une production fruitière. Ainsi, au sein de ce verger conservatoire, la fonction productive initiale est associée au rôle de préservation de la biodiversité domestique. 
De ce fait, le jardin potager comme espace acteur de valeurs environnementales et de lutte contre la précarité transcende l'urbain et le rural. L'engagement à travers le potager se fait de manière indifférenciée entre ces deux territoires. Reste à considérer maintenant les pratiques des jardiniers enquêtés et la façon dont ils considèrent la fonction nourricière des jardins.

\section{La diversité des fonctions jardinières}

L'analyse de l'enquête menée auprès des 34 jardiniers amateurs de trois communes du Roannais a permis de mettre en évidence différentes pratiques potagères. Plusieurs catégories de jardins ont été établies : une première regroupant les jardins consacrés uniquement au potager (5) ; une deuxième où le potager représente une surface plus importante que celle destinée à l'ornement (14); et enfin une troisième où, cette fois, la part réservée à l'ornement est plus conséquente que celle du potager (4). Les 11 jardins restant, uniquement réservés à l'ornement, ne seront donc pas abordés ici.

L'analyse de ces catégories a fait émerger deux conceptions différentes de l'espacepotager : l'une comme acteur de l'alimentation (partie 1) et l'autre comme support de loisirs (partie 2). Les jardiniers appartenant à la première correspondent aux deux premières catégories (19 personnes), puisqu'ils privilégient le potager au jardin d'ornement ou en herbe. Ce sont également des personnes qui ont évoqué le rôle nourricier de leur jardin (ou terme relevant du même champ lexical). Concernant la seconde, afin de comprendre si les jardiniers amateurs rencontrés voient dans leur potager un espace ludique, une question ouverte leur demandait les raisons les poussant à avoir un jardin potager. Les réponses ont été réparties entre des choix relevant de la production ${ }^{5}$ ou des loisirs ${ }^{6}$, ou des deux (si leurs motivations résultent de ces deux champs lexicaux). Ainsi, parmi les 23 jardiniers ayant un potager, 11 cultivent en partie pour leurs loisirs et 3 uniquement pour cela, soit plus de la moitié (14).

\section{Le potager par habitude}

On entend par habitude, ce qui relève des pratiques coutumières, de l'acte de faire un jardin potager parce que cela « a toujours été ainsi », «pour la famille», pour « avoir à manger pour l'hiver », pour « avoir des légumes de qualité-frais-locaux ». Les enquêtes et entretiens ont fait émerger cette pratique à la fois dans les jardins individuels, mais également dans les structures collectives.

\footnotetext{
${ }^{5}$ Réponses obtenues : «Economie - Autoproduction - Travail - Qualité des légumes - Consommation ».

${ }^{6}$ Réponses obtenues : «Plaisir - Intéressant - Passer le temps ».
} 
L'enquête auprès des jardiniers individuels ruraux a révélé que les personnes concernées sont généralement âgées (plus de soixante ans) et représente 15 jardiniers de notre échantillon (19 personnes). Elles font un potager car elles le faisaient déjà dans leurs anciens domiciles, qu'elles soient nouvellement arrivées (moins de 5 ans) ou bien installées depuis plus d'une vingtaine d'années. Le jardin a alors une fonction essentielle dans leur quotidien et dans leur alimentation. Les personnes âgées interrogées habitent généralement à deux, voire seules. La production est souvent excédentaire par rapport à leur consommation (14 personnes interrogées). Les jardiniers peuvent alors être amenés à faire des conserves en prévision de l'époque hivernale. S'il n'y a pas de transformation, la production est donnée en premier lieu aux personnes proches géographiquement (les voisins, amis ou famille qui sont de passage au moment de la récolte), puis à la famille ou aux amis qui n'habitent pas dans leur périmètre. De ce fait, le potager renforce ou est constitutif de liens familiaux.

L'espace en herbe, s'il y en a un, autour du potager a rarement une fonction de loisir. Il est davantage destiné à la petite volaille, aux lapins, à faire pousser de la luzerne ou encore à permettre une rotation des cultures, à créer un équilibre de et dans l'espace.

Ainsi, le jardin permet l'autoconsommation : il est nécessaire pour se nourrir (Gojard, Weber, 1995), mais sa taille réduite n'assure qu'une fonction nourricière saisonnière. Cette fonction nourricière est clairement celle des jardins collectifs de Roanne et découle des contextes économiques et sociaux difficiles. L'élue de la ville de Roanne en cahrge des jardin et le président des jardins ouvriers et familiaux parlent même de potager vivrier. Ainsi, les jardiniers des jardins ouvriers du Matelois, au Nord-Est de Roanne, sont majoritairement précaires et âgés (près de $70 \%$ ). Ils font la navette entre leur domicile (parfois localisé en périphérie de Roanne) et leur parcelle, afin de s'alimenter. Sans sous-entendre que seules les personnes âgées cultivent un potager nourricier, ce sont elles qui semblent majoritairement le faire dans un esprit coutumier. Cet esprit coutumier implique une transmission de valeurs et de bonnes pratiques. Etant principalement le fait de personnes âgées, elles sont les plus à même de renseigner les nouveaux jardiniers. Cependant, la mobilité accrue conduit à une certaine perte du relationnel à la terre entre les générations. Les plus jeunes consultent plus Internet, que leurs parents et grands-parents (bien que certains l'aient évoqué durant l'enquête).

\section{Le potager ludique : support de loisirs}

Le potager est également abordé, par certains jardiniers, comme un espace support de leurs loisirs; comme un espace où le jardinage devient plus un jeu qu'une nécessité ou une habitude. 
Les jardiniers privilégiant le potager comme support de leurs loisirs ne correspondent pas un profil particulier. Parmi le panel de 14 personnes, il y avait 6 femmes et 8 hommes, âgés de 36 à 89 ans, dont 8 retraités. La surface de leurs potagers est également très variable, allant de 20 à $3000 \mathrm{~m}^{2}$. Seules trois personnes se sont installées dans la commune il y a moins de cinq ans. On retrouve cette même diversité chez les 3 personnes ne se consacrant au potager que pour leurs loisirs7. Bien qu'ils jardinent pour leur plaisir, 8 de ces 14 jardiniers consomment la majorité de leur production et la plupart sont souvent, voire tout le temps, dans leur jardin (12 la semaine et 11 le weekend).

Ces jardiniers, bien qu'ils associent leur jardin potager aux loisirs, consacrent néanmoins du temps, de manière assidue, à cette activité.

En parallèle du jardin potager individuel de loisirs, il existe des structures collectives qui donnent au jardin un statut ludique. C'est le cas notamment du jardin pédagogique de Pouilly-les-Nonains. Créé en 2011, pour une durée de quatre à cinq ans, par l'institutrice de la classe de CE1, il a une vocation plus ludique que nourricière. Ceci est notable dès que l'on s'intéresse à la structure : le jardin ne représente en effet qu'une superficie d'environ $5 \mathrm{~m}^{2}$. Si le jardin possède un caractère temporaire et n'est pas suffisamment grand pour nourrir une classe entière, il constitue néanmoins un support pédagogique permettant aux enfants de voir la croissance des plantes: de la graine au fruit. Le caractère ludique apparaît également dans l'implication des élèves. En amont, les enfants sont sensibilisés par leurs familles (notamment les grands-parents) qui possèdent déjà un jardin. Durant l'école, ils consacrent parfois du temps de récréation au désherbage du jardin. Enfin, en aval, les élèves de l'année passée peuvent suivre leur production en récoltant ce qu'ils avaient planté au printemps dernier. Ainsi, ce jardin semble aller au-delà de ses vertus pédagogiques et éducatives, c'est un espace de transmission (Pluvinage, 2003) et de jeux (Donadieu, Fleury, 2003).

Le jardin potager apparaît ici comme un espace favorable aux loisirs autant pour des espaces individuels, que collectifs. A la fonction purement productive du potager, s'ajoute très souvent celle du ludique.

\section{Le jardin potager, un espace porté par le discours environnementaliste}

Si le jardin potager est un espace nourricier et signe d'indépendance face à l'agroindustrie pour certains, un espace support de loisirs pour d'autres, il est aussi le reflet d'un discours ambiant environnementaliste. Depuis la fin des années 1990, avec le sommet de Rio et l'institutionnalisation du développement durable, tout doit être «écologiquement responsable », y compris le potager.

\footnotetext{
${ }^{7}$ Trois hommes, âgés de 41, 64 et 72 ans, dont les parcelles oscillent entre 50 et $300 \mathrm{~m}^{2}$.
} 
A l'échelle des communes, l'engagement prit par certaines8 de ne plus utiliser de produits phytosanitaires et d'encourager leurs habitants à faire de même (engagement « 0 phyto ») en témoigne.

Au niveau individuel, l'enquête de terrain s'intéressait aux types de traitements utilisés par les jardiniers. La première réaction était généralement de dire que « rien n'est mis » et que « tout est bio ». Or, parmi les 34 personnes interrogées, 9 utilisent uniquement des produits biologiques (fumier, purin d'orties, compost), mais des demandes de précisions font émerger une utilisation de produits phytosanitaires pour 9 personnes supplémentaires. II en ressort une définition de l'AB floue pour beaucoup et l'utilisation de produit phytosanitaire en est un exemple. En ce sens, « la promotion de pratiques plus naturelles ne va souvent pas sans culpabilisation de ceux qui n'y adhèrent pas » (Pluvinage, 2003, p. 148). Cette ambivalence apparaît également au regard d'autres pratiques : la définition du compost et son application (pas évidente chez certains) ou a contrario des usages intégrant le cahier des charges de l'AB. C'est notamment le cas des associations de plantes afin de lutter contre certains insectes. Ainsi, 10 jardiniers, majoritairement âgés, ont des œillets d'inde qui repoussent les insectes de manière naturelle. Cependant, ils ne l'évoquent pas comme faisant partie de leurs « pratiques écologiques » du jardin potager.

Ces contradictions peuvent être étendues à l'extérieur du jardin: ainsi, si 15 personnes ont répondu faire un jardin pour connaitre la provenance de leurs légumes, pour «manger local » ou de qualité ; les achats permettant de compléter la production se font, principalement en grandes et moyennes surfaces (GMS) (7) - où la provenance des légumes peut être lointaine -, au marché (4) et rarement (2) chez un producteur local. Parmi tous les jardiniers interrogés (34) seulement un récupère ses semences d'une année sur l'autre. Les autres les achètent sans distinction en GMS ou en magasins spécialisés, ce qui ne permet pas de connaître la provenance et les traitements des graines.

\section{Conclusion}

A travers l'étude des jardins du Roannais on perçoit tout d'abord l'emprunte des héritages de ce territoire, aujourd'hui en crise, sur les pratiques jardinières. Cela se traduit par la prégnance des structures de jardins collectifs et le dynamisme des jardins partagés découlant de l'ESS. S'ajoute un certain renouveau de la fonction nourricière axée sur des valeurs environnementales et patrimoniales. Ainsi, en plus de valeurs nourricières, le jardin potager est aussi porteur d'actions militantes qui transcendent l'urbain et le rural.

\footnotetext{
${ }^{8}$ Saint-Jean-Saint-Maurice-sur-Loire et Roanne
} 
Plus précisément, les jardins potagers apparaissent partagés entre diverses fonctions : d'un côté un rôle nourricier et de l'autre un espace support de loisirs. La dimension temporelle apparaît à plusieurs échelles. Celle du jardin, où le rapport à la terre impose le caractère saisonnier. Celle des jardiniers, où le potager apparaît comme un marqueur de temps: des pratiques limitées quand on est actif, un investissement supplémentaire au début de la retraite, puis un abandon progressif avec l'âge. Celle encore de la société, où le discours environnementaliste actuel appelle à un changement de pratiques ancrées et héritées.

\section{Bibliographie}

BESANCON LOÏS, 2011, «LES JARDINS OUVRIERS, FAMILIAUX ET PARTAGÉS À ROANNE », RAPPORT DE STAGE DE TERRAIN, MASTER DE GÉOGRAPHIE, UNIVERSITÉ LYON 2.

DONADIEU Pierre, FLEURY André, "Les jardiniers restaurent le monde », Les Carnets du paysage $n^{\circ} 9-10,2003$, p. 148-169.

DUBOST Françoise, 1997 (rééd. de Côté jardins, 1984), Les Jardins ordinaires, L'Harmattan, Paris, $176 \mathrm{p}$.

GOJARD Séverine, WEBER Florence, "Jardins, jardinage et autoconsommation alimentaire », I.N.R.A. Sciences sociales, 1995, $\mathrm{n}^{\circ} 2$

PLUVINAGE Manuel, "Pour une histoire sociale du jardinage ", Les Carnets du paysage, $\mathrm{n}^{\circ} 9-10,2003,139-151 \mathrm{pp}$.

WEBER Florence, 1998, L'Honneur des jardiniers. Les potagers dans la France du $X X^{e}$ siècle, Paris, Belin, $285 p$. 